\title{
Superomedial Pedicle versus Inferior Pedicle in Breast Reduction Surgeries
}

\author{
ASSEM H. KAMEL, M.D.*; AHMED H.F. EL-TAWDI, M.D.**; AHMED M. TOHAMY, M.D.*; \\ QUTAIBAH A. ALKANDARI, M.D.*** and AHMED S. SHARAF, M.B.B.Ch.*
}

The Department of Plastic Surgery, Faculty of Medicine, Assiut University*, Faculty of Medicine, Military Medical Academy, Cairo** and The Department of Plastic, Aesthetic \& Burn Surgery, Ministry of Health, Kuwait***

\begin{abstract}
Background: Choosing an ideal technique for breast reduction is a challenging problem specially when the patient lacks an accurate expression of her desired breast size or shape as in the Egyptian society. This study focused on both objective and subjective evaluation of two commonly practiced techniques in breast reduction; the superomedial pedicle and the inferior pedicle.
\end{abstract}

Methods: Nineteen females submitted for breast reduction in a comparative prospective study, 10 of them underwent a superomedial pedicle mammaplasty while 9 patients underwent an inferior pedicle mammaplasty. All the patients assessed for cosmetic outcomes and their satisfaction before and after the surgery.

Results: There was no statistically significant difference between both pedicles regarding the rate of complications. The late post-operative breast measurements including the vertical meridian and nipple-to-inframammary fold distance showed significant higher values in the inferior pedicle group. Also, the rate of boxy-shaped and bottoming out deformity was significantly higher in the same group. Post-operative patient satisfaction showed some higher scores in the superomedial group.

Conclusion: The superomedial pedicle has the advantages of better breast shape and contour and higher patient satisfaction.

Key Words: Superomedial - Pedicle - Breast reduction - Surgeries.

\section{INTRODUCTION}

The female breast is one of the most attractive aesthetic areas in female anatomy. The size, shape, and symmetry of the breasts can have a dramatic effect on the women's well-being. Reduction mammaplasty is certainly one of the operations; plastic surgeons can significantly contribute to a woman's quality of life [1].
Patients requesting reduction present with a variety of problems; from young girls with juvenile hypertrophy and disproportionate breasts to elderly women with large, uncomfortable, sagging breasts with associated breast pain and skin irritation. Understanding patients' motivations is crucially important in deciding which patients are good candidates for this operation [2].

Most cases in our society have moderate to severe mammary hypertrophy who are symptomatic and plagued with back pain and postural changes and who are primarily interested in relieving symptoms, secondarily interested in improving aesthetics, and are less concerned with the amount and location of the scars. These patients typically require a functional rather than cosmetic reduction mammaplasty. And that's why selection of breast reduction technique should be primarily relying on safety \& robust blood supply.

The evaluation of the surgical results via clinical parameters, photographic analysis, and history of complications is considered part of the plastic surgeon's routine. However, in plastic surgery, a surgical specialty dedicated to the improvement of appearance and function, survey instruments that consider the patient's opinion provide valuable information about the effectiveness of surgical interventions. One of these instruments is the Breast- $Q$ which is designed to evaluate outcomes of different breast surgeries including breast reduction [3].

Since its inception in 2006, the BREAST-Q has been used to study breast surgery, providing meaningful and reliable information regarding Health related quality of life and patient satisfaction when used in clinical practice, surgical research, and 
quality improvement initiatives. Use of the BREAST-Q will continue to expand as health related quality of life and patient satisfaction become increasingly important as metrics for evaluating patient care from clinical, policy, and research perspectives [4].

We selected for our study two well-known and safe breast reduction techniques; the superomedial and inferior pedicles, and we conducted a prospective randomized comparative study with 6 months follow-up to evaluate the cosmetic outcomes and patient satisfaction in the Egyptian society.

The inferior pedicle technique has been first described by Ribeiro in 1975 [5] with the NAC being carried on a dermal pedicle, and it is probably one of the most popular breast reduction techniques currently in use in the United States. Advantages of this are well known. It is a rapid and safe technique that can be used on almost every breast size. It has been shown to be as good as or better than other techniques in the preservation of the neurovascular supply to the nipple. It is easily taught and learned [6].

The superomedial pedicle technique was first described by Orlando \& Gutherie in 1975 [7] as a modification of the superior pedicle technique. In this technique the NAC is transposed on a superomedial de-epithelialized pedicle which contains a thin layer of subcutaneous tissue to protect the dermal blood supply. This serves the situation well because it conserves the NAC and breast tissue as a composite block which can be transposed very easily without any tethering. It affords great flexibility when it comes to sculpting the pedicle and varies the lateral tissue resection. In addition, it offers a nice shape to the cleavage of the breast with superior pole fullness [8].

\section{PATIENTS AND METHODS}

This study was conducted on 19 female patients presented with bilateral breast hypertrophy and were submitted for breast reduction in Plastic Surgery Department Assiut University Hospital and Egyptian Military Hospitals in the period from January 2016 to March 2018 and all the patients were categorized in a random fashion into two groups; Group (A): Including 10 cases submitted for superomedial pedicle breast reduction using Hall-Findlay [9] technique and Group (B): Including 9 cases submitted for inferior pedicle breast reduction using the Classic Inverted-T technique [10].
Assessment of our patients pre-operatively \& postoperatively included both objective and subjective methods as follows: Objective assessment was done by means of breast measurements including Vertical Meridian (VM) and Nipple to Inframammary Fold Distance (Ni-IMF), incidence of early complications e.g. hematoma, seroma, congestion/ischemia of the nipple areola complex and wound dehiscence, late follow-up evaluation after 6 months regarding overall symmetry, breast shape $\&$ contour, recurrence of ptosis.

Subjective assessment by evaluating patient satisfaction using an Arabic translated valid version of Breast-Q Reduction/Mastopexy Module which was self-applied by the patient preoperatively and post-operatively.

In our study we used the Breast-Q pre-operative reduction module including three subthemes which are, satisfaction with breast, psychological wellbeing and physical well-being. At follow-up we used the post-operative reduction module including 5 subthemes, satisfaction with breast, psychological well-being, physical well-being, satisfaction with outcome and satisfaction with nipples. All BreastQ scores computed from the responses to the separate questions by adding them together and converting the score to a scale from 0 to 100 (similar to conversion into a percentage). A higher score means high satisfaction or better health-related quality of life.

\section{RESULTS}

This study included total number of 19 patients, 10 of them underwent superomedial breast reduction (Group A) and 9 of them had inferior pedicle breast reduction (Group B). All of them are nonlactating females or stopped lactation at least 12 months before surgery. patients' demographic data demonstrated in (Table 1) and there was no significant difference between patient demographics of the two studied groups pre-operatively.

Regarding pre-operative Breast measurements, in Group (A), pre-operative (VM) had been measured and ranged from $25-41 \mathrm{~cm}$. While in Group (B) it was ranged from $28-41 \mathrm{~cm}$. The Ni-IMF in Group (A), ranged from $12-20 \mathrm{~cm}$. While in Group (B) it was ranged from $13-23 \mathrm{~cm}$. All the patients in both groups were beyond 3rd degree of ptosis. There was no significant difference between breast measurements of the two studied groups preoperatively. 
There was no statistically significant difference between Group (A) and Group (B) regarding the rate of post-operative complications. In group (A) wound infection occurred in one case $(10 \%)$, decreased nipple sensation in one case $(10 \%)$, minor wound dehisence in two cases $(20 \%)$, no NAC necrosis with just congestion in two cases (20\%), no hematoma or seoma formation. In Group (B) no wound infection occurred, wound dehisence in two cases $(22 \%)$, no NAC necrosis with just congestion in one case $(11 \%)$, no permanent decreased nipple sensation, no hematoma or seroma formation.

The late post-operative breast measurements after 6 months in our study showed a highly significant difference between breast measurements of the two studied groups post-operatively ( $p$-value $<0.01$ ) with the VM and Ni-IMF distances being longer in Group (B). In Group (A) none of the cases develop ptosis postoperatively. While in group (B) one case (11\%) develop 1st degree ptosis (Table 2).

Regarding the cosmetic outcomes in our study, in Group (A), one case (10\%) had mild asymmetry in NAC position without need for revision. One case $(10 \%)$ had superior malposition of NAC needing revision, all cases had sound rounded contour and one case (10\%) had bottoming out deformity. While in Group (B), 2 cases (22\%) had mild asymmetry in NAC position without need for revision, one case (11\%) had mild asymmetry in NAC size without need for revision, 4 cases $(44 \%)$ had boxyshaped breast. 5 cases $(55 \%)$ had bottoming out deformity. There was a significant difference between incidence of boxy-shape deformity \& bottoming out deformity in both groups ( $p$-value $<0.05)$.

The assessment of patient satisfaction in our study showed no statistically significant difference between Group (A) and Group (B) as regard preoperative Breast-Q scores including three modules; satisfaction with breast, psychological well-being and physical well-being. While in post-operative patient satisfaction, there was a highly significant difference between Group (A) and Group (B) ( $p$ value $<0.01$ ) in two modules which are satisfaction with breast and satisfation with the nipples being with higher scores in superomedia Group (A) (Tables 3,4).
Table (1): Comparison of patient characteristics between Group (A) and Group (B).

\begin{tabular}{llllc}
\hline & $\begin{array}{c}\text { Total } \\
(\text { Mean } \pm \text { SD) }\end{array}$ & $\begin{array}{c}\text { Group (A) } \\
(\text { Mean } \pm \text { SD) }\end{array}$ & $\begin{array}{c}\text { Group (B) } \\
(\text { Mean } \pm \text { SD) }\end{array}$ & $\begin{array}{c}p- \\
\text { value }\end{array}$ \\
\hline Number of patients & 19 & 10 & 9 & - \\
Age, years & $30.4 \pm 4.63$ & $30.6 \pm 3.74$ & $30.11 \pm 5.18$ & 0.8145 \\
Weight, Kg & $86.6 \pm 13$ & $82.9 \pm 12.43$ & $90.77 \pm 13.08$ & 0.1965 \\
BMI, Kg/M 2 & $32.3 \pm 6$ & $36.62 \pm 5.7$ & $33.08 \pm 6.58$ & 0.2258 \\
Hb level, g/dl & $13 \pm 1$ & $12.93 \pm 0.98$ & $13.7 \pm 1.06$ & 0.1182 \\
\hline
\end{tabular}

Table (2): Comparison of post-operative breast measurements between Group (A) \& Group (B).

\begin{tabular}{llll}
\hline & Group & Mean \pm SD & $p$-value \\
\hline Rt. VM & Group A & $19.7 \pm 0.67$ & $* 0.0002$ \\
& Group B & $21 \pm 0.5$ & \\
Lt. VM & Group A & $19.8 \pm 0.57$ & $* 0.0001$ \\
& Group B & $21.2 \pm 0.63$ & \\
Rt. Ni-IMF & Group A & $11.6 \pm 1.17$ & $* 0.0003$ \\
& Group B & $13.55 \pm 0.52$ & \\
Lt. Ni-IMF & Group A & $11.7 \pm 1.16$ & $* 0.0007$ \\
& Group B & $13.44 \pm 0.52$ & \\
\hline
\end{tabular}

Table (3): Comparison of pre-operative Breast-Q scores between Group (A) \& Group (B).

\begin{tabular}{llll}
\hline & Group & Mean \pm SD & $p$-value \\
\hline Satisfaction with breasts & Group A & $18.77 \pm 9.61$ & 0.4262 \\
& Group B & $15.11 \pm 9.95$ & \\
Psychosocial well-being & Group A & $31.44 \pm 8.59$ & 0.5644 \\
& Group B & $29 \pm 9.51$ & \\
Physical well-being & Group A & $50.66 \pm 8.15$ & 0.0634 \\
& Group B & $43.77 \pm 6.81$ & \\
\hline
\end{tabular}

Table (4): Comparison of post-operative Breast-Q scores between Group (A) \& Group (B).

\begin{tabular}{llll}
\hline & Group & Mean \pm SD & $p$-value \\
\hline Satisfaction with breasts & Group A & $73 \pm 4.21$ & $* 0.0088$ \\
& Group B & $64.77 \pm 7.61$ & \\
Psychosocial well-being & Group A & $75.66 \pm 5$ & 0.2543 \\
& Group B & $72.22 \pm 7.58$ & \\
Physical well-being & Group A & $77 \pm 8.48$ & 0.0805 \\
& Group B & $70.44 \pm 6.67$ & \\
Satisfaction with outcome & Group A & $80.77 \pm 10.57$ & 0.6863 \\
& Group B & $78.88 \pm 9.34$ & \\
Satisfaction with Nipples & Group A & $83.44 \pm 9.19$ & $* 0.0036$ \\
& Group B & $70.66 \pm 7.03$ & \\
\hline
\end{tabular}




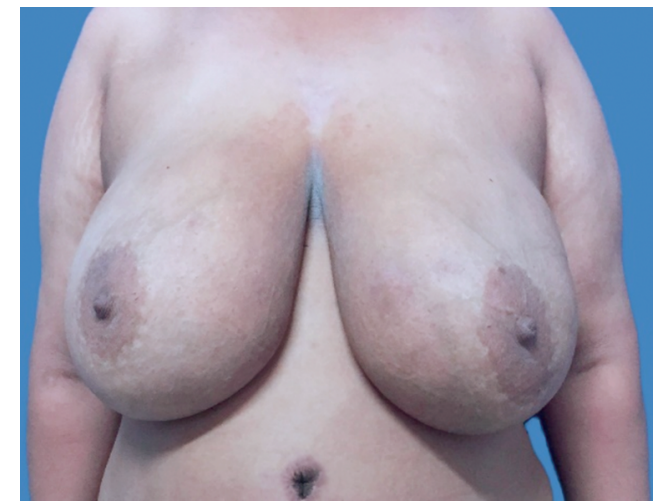

(A): Pre-operative anterior view.

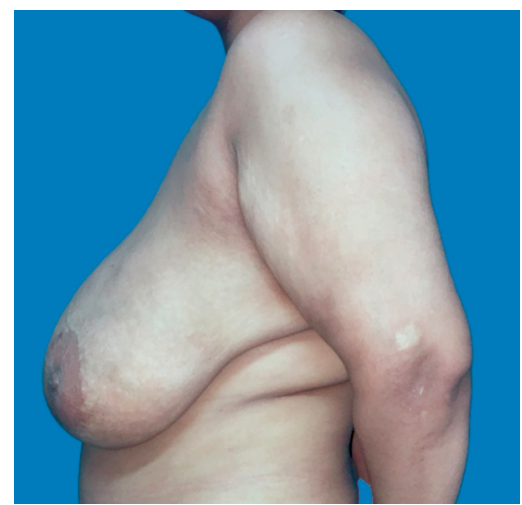

(C): Post-operative anterior view.

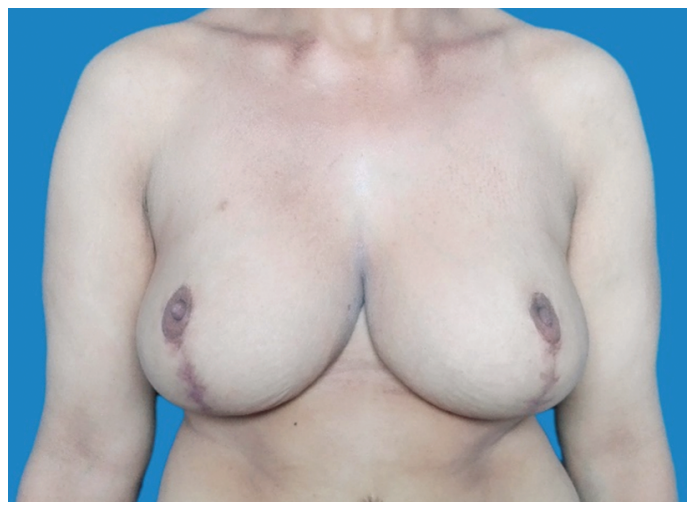

(B): Pre-operative left lateral view.

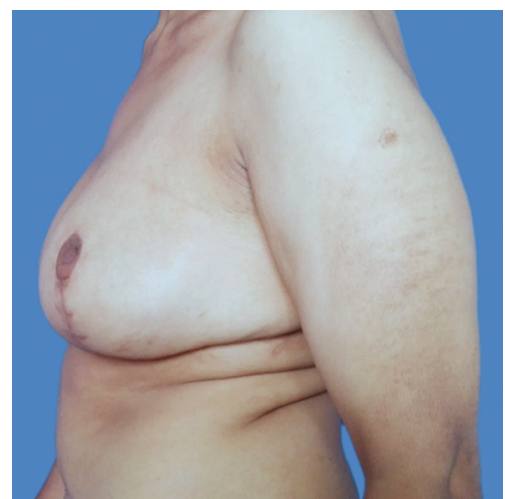

(D): Post-operative left lateral view.

Fig. (1): Pre-operative (above) and 6 months post-operative (below) photographs of a 30 years old woman with $32 \mathrm{~cm} \mathrm{Rt.} \mathrm{VM} \mathrm{\&} 34 \mathrm{~cm} \mathrm{Lt}$. VM operated with Inferior pedicle inverted-t mammaplasty.

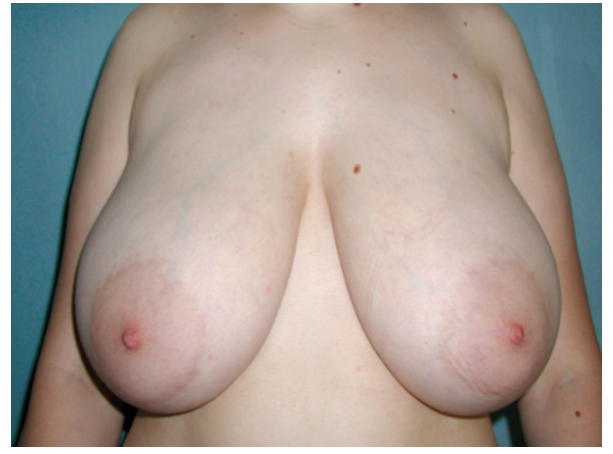

(A): Pre-operative anterior view.

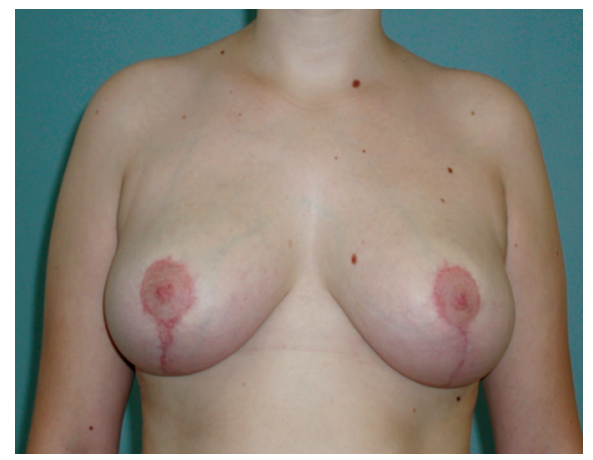

(C): Post-operative anterior view.

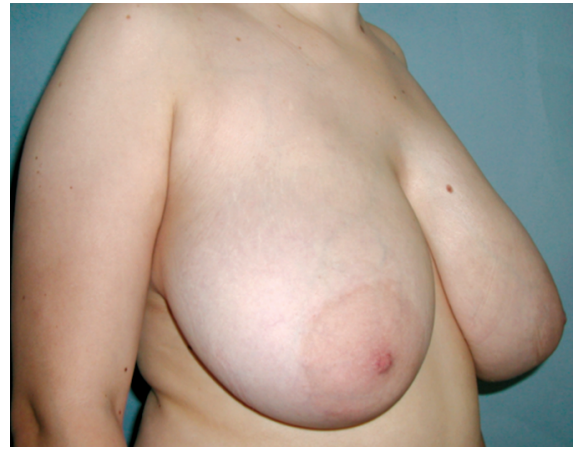

(B): Pre-operative right oblique view.

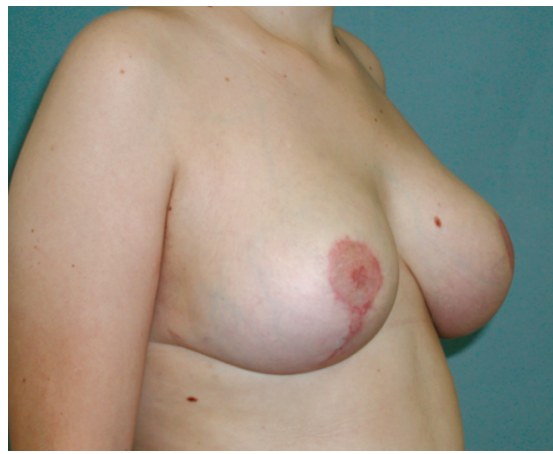

(D): Post-operative right oblique view.

Fig. (2): Pre-operative (above) and 5 months post-operative (below) photographs of a 38 years old woman with $30 \mathrm{~cm}$ VM operated with superomedial vertical scar mammaplasty. 


\section{DISCUSSION}

The evolution of reduction mammaplasty over the past century can be characterized as innovative, imaginative, and diverse. It is estimated that over 50 variations and techniques for reduction mammaplasty have been described. These variations are based on skin pattern design, as well as pedicle selection for transposition of the nipple areolar complex. Skin patterns have varied. from short scar to long scar techniques. Nipple transposition techniques have varied from free graft to dermal pedicles to dermo-parenchymal pedicles with orientations that can be anywhere along a $360^{\circ}$ circle. All of these approaches have demonstrated success in certain situations. The debate as to whether one technique for reduction mammaplasty would be sufficient for all types of patients and breasts has been abandoned for all intent and purposes. It is accepted that individualized options for reduction mammaplasty are preferred and will usually generate optimal outcomes [11].

Our study had selected two groups of patients, both could be candidates for many breast reduction techniques. All of them had moderate to large volume breasts with 3 rd degree ptosis. Most of them were with expected breast tissue resection approximately 1000 grams from each side.

Analysis of the complications associated with different techniques may provide a clue to their relative success. At the same time, it is very difficult to compare the outcome of different techniques for reduction mammaplasty, as the variables of body build, breast size, degree of reduction achieved, skin elasticity, distance of transposition of the NAC, and other patients varies [1].

According to a recent study conducted by Kemaloglu \& Özocak [12] which compared outcomes of inferior and superomedial pedicle technique in patients with gigantomastia, there was no statistically significant difference in either major or minor complications between the 2 groups. In the inferior pedicle group, partial nipple necrosis occurred in $4 \%$ of patients, wound dehisence in $8 \%$, and decreased nipple sensation in $4 \%$ ). In the superomedial pedicle group, no major complications occurred, wound dehisence in $4 \%$, and decreased nipple sensation in $4 \%$ ).

The complication rate in our study is keeping with previous studies which show rates ranging from $7 \%$ up to $63 \%$ with only $6 \%$ require surgical intervention $[\mathbf{1 3}, \mathbf{1 4}]$. There was no statistically significant difference between Group (A) and
Group (B) as regard the rate of post-operative complications.

As regards functional outcome of breast reduction, it includes NAC sensation and breast lactation. Assessment of breast lactation wasn't feasible at our study as it requires late follow-up at least a year after surgery and also many of the patients seeking breast reduction are either non-married or multiparous with no willings for further pregnancies. Also our findings in breast sensation were limited by time factor as the maximum late followup in our study was at 6 months while the sensory changes can be improved even after 12 months.

Regarding breast measurements and according to a study conducted by Zhu et al., [15], the inferior pedicle breasts demonstrated increased lengthening of the sternal notch-to-nipple distance and the NiIMF distance when compared with the superomedial pedicle. Our study confirmed these findings as there was a highly significant difference between Group (A) and Group (B) with the postoperative $\mathrm{VM}$ and Ni-IMF distance both being longer in inferior pedicle group most propably due to the more bottoming out and recurrent ptosis associated with the inferior pedicle technique.

According to a study by Makboul et al., [16], the superomedial pedicle shows better long term aesthetics, projection and contour of the breast in addition to the less incidence of glandular ptosis. Our study confirmed these findings as there was a statistically significant difference between Group (A) and Group (B). The rate of bottoming out and boxy-shape deformities is much higher in the inferior pedicle group (Fig. 1) in contrast with the superomedial group which had better breast shape, projection and contour (Fig. 2).

Regarding NAC position and symmtery, it was concluded by Altuntaş et al., [17] that both downward or upward transposition of NAC are directly proportional with the amount of residual breast volume, skin quality, skin envelope and body weight changes rather than the type of pedicle used. In our study there was no statistically significant difference between Group (A) and Group (B) in the NAC position or symmetry.

Our prospective study confirms what has been previously reported in the literature regarding overall improvement in patient satisfaction using Breast-Q score. As according to a previous prospective study conducted by Coriddi et al., [18] and also a recent study by Cabral et al., [19], there was a statistically significant improvement in physical and psychosocial well-being after breast reduction. 
Also, patients are generally satisfied with the new appearance of their breasts, which are smaller and lifted.

But it was the first time to impliment the BreastQ to compare patient satisfaction between superomedial pedicle and inferior pedicle breast reduction in egyptian patients. We also found a highly significant difference between Group (A) and Group (B) in two of the post-operative modules which are satisfaction with breast and satisfation with the nipples being with higher scores and consequently better satisfaction in superomedial group.

\section{Conclusion and Recommendations:}

Both superomedial and inferior pedicle techniques are safe, reliable and applicable on Egyptian patients for breast reduction. And the own surgeon experience is the main factor which affects his choice between both pedicles. We also proved the advantages of superomedial pedicle as regard Breast projection and contour, less incidence of Bottoming-out phenomena and higher patient satisfaction about the outcome.

Also we recommend furher studies on both pedicles regarding recurrent ptosis and late contour using three dimensional analysis for accurate measurements and assessment. And application of Breast-Q score on a regular basis for evaluation of any patient undergoing cosmetic breast surgery.

\section{REFERENCES}

1- Fahmy F.S. and Saleh M.A.A.: Reduction Mammoplasty:"The Piece of Art". In: Shiffman M.A., editor. Cosmetic Surgery Art and Techniques. Berlin: Springer, p. 755-74, 2013.

2- Jones G.E. and Hall-Findlay E.J.: Reduction Mammaplasty. In: Jones GE, editor. Bostwick's plastic \& reconstructive breast surgery. New York: Thieme Publishers, 2010.

3- Cabral I.V., Da Silva Garcia E., Sobrinho R.N., Pinto N.L.L., Juliano Y., Veiga-Filho J., Ferreira L.M. and Veiga D.F.: Use of the BREAST-QTM Survey in the Prospective Evaluation of Reduction Mammaplasty Outcomes. Aesthetic Plastic Surgery, 42 (2): 388-95, 2018.

4- Cohen W.A., Mundy L.R., Ballard T.N., Klassen A., Cano S.J., Browne J. and Pusic A.L.: The BREAST-Q in surgical research: A review of the literature 2009-2015. Journal of Plastic, Reconstructive \& Aesthetic Surgery, 69 (2): 149-62, 2016

5- Ribeiro L.: A new technique for reduction mammaplasty. Plastic and Reconstructive Surgery, 55 (3): 330-4, 1975.

6- Maxwell G.P. and White D.J.: Inferior pedicle technique of breast reduction. Operative Techniques in Plastic and Reconstructive Surgery, 3 (3): 170-5, 1996.
7- Orlando J. and Guthrie R.: The superomedial dermal pedicle for nipple transposition. British Journal of Plastic Surgery, 28 (1): 42-5, 1975.

8- Antohi N., Isac C., Bratu T., Vitalie S. and Florian B.: Breast Reduction/Mastopexy with Superomedial Pedicle. In: Mugea TT, Shiffman MA, editors. Aesthetic Surgery of the Breast. Berlin: Springer, p. 819-31, 2015.

9- Hall-Findlay E.J.: A simplified vertical reduction mammaplasty: Shortening the learning curve. Plastic and Reconstructive Surgery, 104 (3): 748-59, 1999.

10- Wise R.J.: A preliminary report on a method of planning the mammaplasty. Plastic and Reconstructive Surgery, 17 (5): 367-75, 1956.

11- Nahabedian M.Y.: Reduction mammaplasty with invertedT techniques. In: Nahabedian MY, Neligan PC, editors. Plastic Surgery E-Book: Volume 5: Breast: Elsevier Health Sciences, p. 136-59, 2017.

12- Kemaloglu C.A. and Özocak H.: Comparative Outcomes of Inferior Pedicle and Superomedial Pedicle Technique With Wise Pattern Reduction in Gigantomastic Patients. Annals of Plastic Surgery, 80 (3): 217-22, 2017.

13- Cunningham B.L., Gear A.J., Kerrigan C.L. and Collins E.D.: Analysis of breast reduction complications derived from the BRAVO study. Plastic and Reconstructive Surgery, 115 (6): 1597-604, 2005.

14- Winter R., Haug I., Lebo P., Grohmann M., Reischies F.M., Cambiaso-Daniel J., Tuca A., Rienmüller T., Friedl H. and Spendel S.: Standardizing the complication rate after breast reduction using the Clavien-Dindo classification. Surgery, 161 (5): 1430-5, 2017.

15- Zhu V.Z., Shah A., Lentz R., Sturrock T., Au A.F. and Kwei S.L.: A Comparison of Superomedial versus Inferior Pedicle Reduction Mammaplasty Using Three-dimensional Analysis. Plastic and Reconstructive Surgery, 138 (4): 781e-3e, 2016.

16- Makboul M., Abdelhamid M.S. and Al-Attar G.S.: Longterm follow-up and patient satisfaction after reduction mammoplasty: Superomedial versus inferior pedicle. Indian Journal of Plastic Surgery: Official Publication of the Association of Plastic Surgeons of India, 49 (2): 214, 2016.

17- Altuntaş Z.K., Kamburoğlu H.O., Yavuz N., Dadacı M. and İnce B.: Long-Term Changes in Nipple-Areolar Complex Position and Inferior Pole Length in Superomedial Pedicle Inverted 'T'Scar Reduction Mammaplasty. Aesthetic Plastic Surgery, 39 (3): 325-30, 2015.

18- Coriddi M., Nadeau M., Taghizadeh M. and Taylor A.: Analysis of satisfaction and well-being following breast reduction using a validated survey instrument: The BREAST-Q. Plastic and Reconstructive Surgery, 132 (2): 285-90, 2013.

19- Cabral I.V., Da Silva Garcia E., Sobrinho R.N., Pinto N.L.L., Juliano Y., Veiga-Filho J., Ferreira L.M. and Veiga D.F.: Use of the BREAST-Q ${ }^{\mathrm{TM}}$ Survey in the Prospective Evaluation of Reduction Mammaplasty Outcomes. Aesthetic Plastic Surgery, 1-8, 2017. 